ESTIMATION DE PARAMETRES DISTRIBUES

DANS DES EQUATIONS AUX DERIVEES

PARTIELLES

\title{
G. CHAVENT
}

(Institut de Recherche d'Infornatique et d'Automatique et Université Paris IX - Dauphine)

La méthode présentée ici est conçue spécialement pour 1 'estimation de paramètres distribués dans des systèmes distribués.Elle utilise la théorie moderne du contrôle dans des espaces de fonctions, et consiste à minimiser un critère d'erreur (non quadratique en les paramètres) par une méthode de gradient ordinaire. Les avantages de cette façon de procéder sont les suivants :

- il n'est pas besoin de supposer que 1 'on connaît une forme algébrique des fonctions paramètres pour calculer le gradient par rapport à cette fonction.

- Iorsqu'on désire tester diverses formes algëbriques (chacune ne contenant que quelques paramètres inconmus), il suffit de modifier quelques cartes dans le programme, dont 1'architecture reste inchangêe.

- le calcul de ce gradient ne nécessite que la résolution de deux équations aux dérivées partielles, même lorsque l'inconnue est une fonction.

- la méthode est utilisable même si l'on ne possède que peu de points de mesures en temps ou en espace

Nous donnons des applications à l'estimation de fonctions des variables d'espace (dans des nappes d'eau et de pétrole) et à l'identification d'une fonction dépendant de la solution du système.

Communication présentée au Colloque International sur les Méthodes de Calcul Scientifique et Technique I.R.I.A., Ie Chesnay, 17-21 décembre 1973. 


\section{I - INTRODUCTION}

Le problème de l'estination des paramètres peut être posé comme un problème de contrôle optimal dans lequel le contrôle serait le paramètre à estimer, et où 1a fonction coût serait un critère quadratique d'erreur entre les mesures et les sorties calculées par le modèle. Il êtait donc naturel de transposer à l'estimation. de paramètres distribués dans des systèmes distribués les mêthodes d'analyse fonctionnelle développées par J.L. LIONS ( $[1],[2]$ ) pour le contrôle des systèmes distribués. La différence principale est que, dans notre cas, la fonctionnelle: minimiser n'est pes quadratique, de sorte que nombre de résultats de la théorie ne peuvent être transposês. Mais cette approche nous donne un algorithme de calcul du gradient, qui est systèmatique et efficace.

Au $\S I I$, nous rappelons quelques notations d'analyse fonctionnelle; Au $\S I I$, nous esquissons la théorie; Enfin le SIV donne trois applications numériques.

\section{II - NOTATIONS}

$X$ et $Y$ étant deuX espaces de Banach, on notera:

$X^{\prime}$ et $Y^{\prime}$ les espaces duaux topologiques de $X$ et $Y$

$\mathcal{L}(X, Y) I^{\prime}$ espace des applications linéaires continues de $X$ dans $Y$

\|\|$_{X}$ et \|\|$_{Y}$ les normes dans les espaces $X$ et $Y$ si $A \in \mathcal{L}(X, Y)$ on notera par $A^{*} € \mathcal{L}\left(Y^{\prime}, X^{\prime}\right)$ le transposé de $A$

$(,)^{\prime} X$ sera utilisée pour la dualité entre $X$ ' et $X$

()$_{X}$ sera utilisée pour le produit scalaire dans $X$

(1orsque X est un Hilbert naturellement).

Si $\Omega$ est un ouvert de $R^{\mathrm{n}}$, on introduit les espaces de fonctions sur $\Omega$ suivants :

$C(\Omega)=$ espace des fonctions continues sur $\Omega$

$L^{\infty}(\Omega)=$ espace des fonctions p.p. bornées sur $\Omega$

$\mathrm{L}^{2}(\Omega)=$ espace des fonctions de carré Lebesgue intégrable

$H^{1}(\Omega)=\left\{u \in L^{2}(\Omega) \mid \frac{\partial u}{\partial x_{i}} \in L^{2}(\Omega) i=1,2 \ldots n\right\}$

$H_{0}^{1}(\Omega)=\left\{u \in H^{1}(\Omega) \mid " u=0\right.$ sur le bord $\Gamma$ de $\left.\Omega^{\prime \prime}\right\}$.

Dans tout le reste de 1'article, on écrira dérivable pour Fréchet-Dérivable.

\section{III - IHEORIE}

Supposons que les equations gouvernant notre système soient de forme connue. Seuls sont inconnus, (ou mal connus) un ensemble de parametres, qui peuvent être 
des nombres ou des fonctions.

L'équation d'ëtat sera :

$$
\psi(y, a)=f
$$

où (Y, Q et F êtant des Banach) :

$$
\begin{aligned}
& \text { y } \in \mathrm{Y} \text { est } 1 \text { 'état du système } \\
& \text { a } \in \text { Qest } 1 \text { 'ensemble des paramètres inconnus } \\
& \mathrm{f} \in \mathrm{F} \text { est } 1 \mathrm{e} \text { 2ème membre de l'équation d'état } \\
& \psi \text { est une application connue de } \mathrm{Yx} \mathrm{C} \longrightarrow \mathrm{F} \text {. }
\end{aligned}
$$

Nous supposerons qu'il existe une partie ouverte $a_{c}<a_{\text {telle que : }}$

Pour tout a $\in a_{c}$, l'équation (1) a une solution et une seule $y \in Y$.

Nous noterons cette solution $y(a)$.

Remarque__l : Le temps n'apparaît pas explicitement dans 1'équation (1). Cependant, $Y$ peut être un ensemble de fonctions du temps (cf par exemple les applications II et III) ; l'équation (1) contient donc aussi les systèmes dynamiques.

Pour estimer le paramètre $a$, il nous faut quelques informations supplémentaires sur le système (1). Introduisons pour cela un espace de Hilbert $H_{b}$, qui sera notre espace des observations, et une application $\ell$ de $Y$ dans H. Bien que la théorie puisse être faite sans difficulté pour $\zeta$ non linéaire, mais continument dérivable, nous supposerons dans la suite pour des raisons de simplicité:

$$
e \in \mathscr{Z}(Y ; 1)
$$

Appelons $a_{0} \Leftarrow a_{c}$ la valeur exacte, mais inconnue des paramètres. L'observation consiste alors en un certain élément

$$
\left.\begin{array}{l}
z \in \mathcal{H}, \text { supposé être une "mesure" } \\
\text { de } \zeta_{\mathrm{y}\left(\mathrm{a}_{\mathrm{o}}\right)}
\end{array}\right\}
$$

On peut alors associer à tout paramètre a $\leqslant a_{c}$ une fonction coût $J(a)$ par :

$$
J(a)=\left\|e_{y(a)-z}\right\|_{f \in}^{2} \forall a \in a_{c}
$$


Remargue_2 : Si l'on considère l'observation comne la sortie du système, cette fonction coût n'est rien d'autre que l'écart quadratique entre les sorties calculées et mesurées.

Nous pouvons alors formuler ainsi notre problème didentification :

$$
\begin{aligned}
& \text { trouver â } \in C_{a d} \text { tel que : } \\
& J(\hat{a}) \leqslant J(a) \quad \forall a \in a_{a d}
\end{aligned}
$$

où

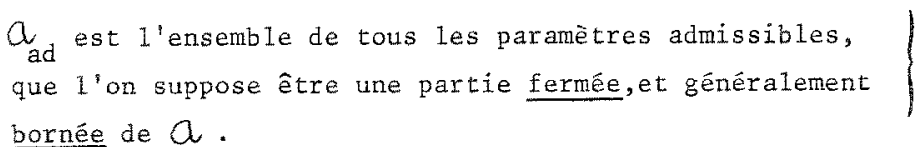
bornée de $a_{c}$.

On peut alors se poser au moins trois questions :

i) Sous quelles conditions le problème (6) a-t-il au moins une solution ?

ii) Sous quelles conditicns le problème (6) a-t-iI au plus une solution ?

iii) Comment effectivement minimiser $J$ ?

Il n'y a pas de réponse géteêrale à la question i), tout au moins lorsque l'espace $a$ des paramètres est de dimension infinie. Lorsque $a$ est de dimension finie, on a le résultat suivant (trivial).

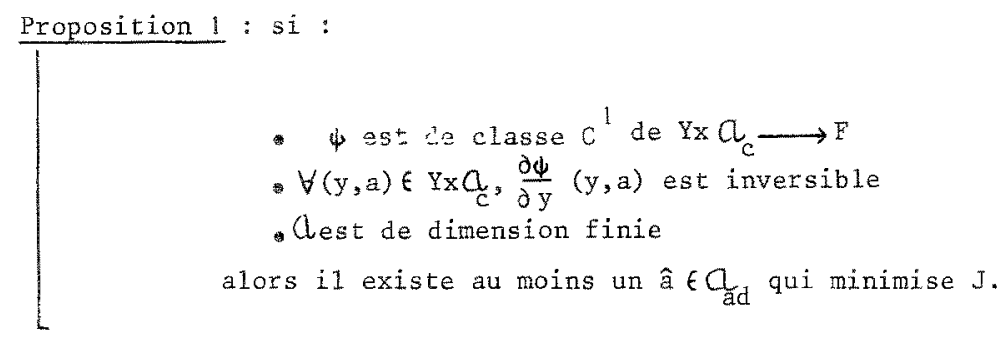

Démonstration : (2) (3) (8) et (9) et le théorème des fonctions implicites montrent que $J$ est continue sur $a_{c}$, et (7) et (10) entraînent que $a_{a d}$ est compact. La question ii) est importante du point de vue des applications. En effet, s'il n'y a qu'une solution â au problème (6), on peut espérer que, sous des hypothèses raisonnables, â sera "voisin" de $a_{0}$ si z est "voisin de Gy(a $\left.a_{0}\right)$. Malheureusement, il n'y a là encore aucun résultat général d'unicité. Le cas gênéral serait plutôt le cas de non-unicité. Pour avoir alors une idée de la confiance que l'on peut avoír en $\hat{a}$, on minimise $J$ à partir de diverses valeurs 
initiales.

En ce qui concerne la question $\mathrm{iii),} \mathrm{nous} \mathrm{avons} \mathrm{heureusement} \mathrm{des} \mathrm{résultats} \mathrm{qui}$ nous permettront de calculer numëriquement â, même en 1 'absence de résultats théoriques d'existence et ou d'unicitê.

La minimisation de $J$ se fera par une méthode de gradient. Nous avons utilisê Ia mêthode de steepest-descent; En effet, la fonctionnelle J n'étant absolument pas quadratique, il n'est pas sûr que des méthodes plus élaborées (celles du gradient conjugué, ou de Fletcher Powel1), utilisant le caractère supposé quadratique de la fonctionnelle, donnent un gain important (un test fait dans [5] entre steepestdescent et gradient conjugué les a mis à êgalité. Mais si on le dêsire, n'importe quelle autre mêthode utilisant le gradient peut être utilisée.

$D^{\prime}$ autre part, il faut choisir avec soin le mode de calcul du gradient de $J$. En effet, si a est une fonction, elle peut être représentêe numériquement par sa valeur en de nombreux points, de telle sorte que le gradient de J peut avoir de nombreuses composantes (quelques centaines dans certaines applications) : le calcu1 de ce gradient par différences finies par exemple prendrait un temps prohibitif.

La réponse thêorique est donnée par 1a

Proposition 2: sous les hypothèses (8) et (9), la fonctionnelle J est de classe $\mathrm{C}^{\prime}$ de $a_{\mathrm{c}} \longrightarrow \mathbb{R}$. La dérivée $J^{\prime}(a) \in Q^{\prime}$ est donnée par:

$J^{\prime}(a) \cdot \delta a=\frac{\partial k}{\partial a}(y(a), a) \cdot p \quad \forall a \in a_{c}, \quad \forall \delta a \in C$

où :

- $y(a) \in Y$ est 1 'état du système, solution de (1) avec la valeur a des paramètres inconnus

- $\rho \notin F^{\prime}$ est l'état adjoint, solution de :

$\frac{\partial q^{*}}{\partial y}(y(a), a) \cdot p=-2 b^{*} \Lambda(b y(a)-z)$

où $\Lambda$ représente $1^{\prime}$ isomorphisme canonique de $\not{Z}$ sur son dual $\mathfrak{H}^{\prime}$ '

\section{Démonstration.}

D'après le théorème des fonctions implicites, 1'application a $\rightarrow$ y dêfinie par (1) est dérivable de $a_{c} \rightarrow Y$, la dérivée en a $\in O_{c}$ étant 1'application qui, à $\delta a \in Q$ fait correspondre $\delta$ y $\in$ Y solution de :

$$
\frac{\partial \psi}{\partial y}(y, a) \delta y=-\frac{\partial \psi}{\partial a}(y, a) \cdot \delta a
$$


La différentielle $\delta J$ du critère $j$ défini en $(j)$ correspondant à unz variatior Sa du paramètre s'écrit donc :

$$
\delta \mathrm{J}=2\left(\mathscr{B y}(a)-z, \delta^{\delta} y\right)
$$

c.a.d.

c.a.d.

$$
\begin{aligned}
\delta J & =2(\Lambda(\& y(a)-z), \delta y) \partial b^{\prime}, J b \\
& =2\left(b^{*} \Lambda(\delta y(a)-z), \delta y\right) Y^{\prime} Y \\
\delta J & =-\left(\frac{\partial \psi^{*}}{\partial y}(y, a) \cdot p, \delta y\right)_{Y^{\prime} Y} \\
& =\left(p,-\frac{\partial \psi}{\partial y}(y, a) \cdot \delta y\right)_{F^{\prime} F}
\end{aligned}
$$

ce qui donne bien (II) en utilisant ( 12 bis).

Voyons maintenant comment ce rësultat nous permet pratiquement de calculer le gradient de la fonction J par rapport aux paramētres inconnus a. La formule (11) se rêécrit :

$$
\begin{aligned}
& J^{\prime}(a) \cdot \delta a=\left(\rho, \frac{\partial \Psi}{\partial a}(y(a), a) \cdot \delta a\right)^{\prime} F \\
& \forall a \in a_{c} \text { et } \forall \delta a \in a
\end{aligned} \mid
$$

Nous distinguerons deux cas:

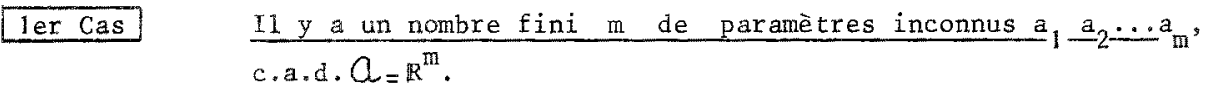

Alors le dual $a$ de $a$ peut être identifié à $a=R^{\text {ma }}$ àde du produit scalaire usuel. Et la formule (13) devient:

$$
\left.\begin{array}{c}
J^{\prime}(a) \cdot \delta_{a}=\sum_{j=1}^{m} \gamma_{j} \delta a_{j} \\
\forall \delta_{a}=\left(\delta a_{1} \ldots \delta a_{m}\right) \in \mathbb{R}^{m}
\end{array}\right\}
$$

Au vu de la formule (14), on obtient les composantes du gradient de $J$ par rapport au vecteur a :

$$
\frac{\partial J}{\partial a_{1}}(a)=\gamma_{1}, \ldots, \frac{\partial J}{\partial a_{m}}(a)=\gamma_{m}
$$


2ème cas Le paramètre inconnu est une fonction a de la variable $u$, prenant ces valeurs dans un domaine borné $D \subset \mathbb{R}^{p}$.

La variable u peut être une des variables indépendantes du problème ( 1 'espace ou le temps par exemple, cf. les applications 1 et 2), mais peut aussi être une des variables dépendantes du problème (1'état du système par exemple, cf. 1'application 3).

Qest alors un espace de dimension infinie et il n'y a alors plus, en gênéral d'application de dualité de $\boldsymbol{Q}$ surson dual $\boldsymbol{a}^{\prime}$. Prenons par exemple le cas suivant :

$$
a=8(D)
$$

Alors le gradient de J par rapport à a est dans C', qui est ici l'espace des mesures de Radon sur $D$, qui est bien plus "grand" que $I$ 'espace des fonctions continues sur D!

Heureusement, il se trouve que dans les applications pratiques, 1a mesure J'(a) est de la forme $\gamma(u)$ du, où $\gamma$ est une fonction Lebesgue intégrable sur D. Dans ce cas, la formule (13) devient :

$$
J^{\prime}(a) \delta a=\int_{D} \gamma(u) \delta a(u) d u \quad \forall \delta a \in a
$$

(cette formule est à comparer avec la formule (14) : la somme discrēte a étê remplacée par une intégrale).

Cette fonction $\gamma(u)$, lorsqu'elle existe, s'appelle la dérivêe partielle fonctionnelle de J par rapport à la fonction a :

$$
\frac{\partial J}{\partial a}(u)=\gamma(u) \quad \forall u \in D
$$

(à comparer avec (15)).

Dans les deux cas, la proposition 2 nous permet de calculer d'un seul coup 1e gradient $J$ par rapport à a (que ce gradient soit un vecteur $\left(\gamma_{1} \ldots \gamma_{\mathrm{m}}\right)$ ou une fonction $\gamma(u)$ ) à l'aide de $y, \rho$ et de la formule (13). Le calcul numérique de ce gradient ne nécessite donc que deux résolutions de systèmes : 1'équation directe (1), I'équation adjointe (12), cette dernière étant toujours linéaire.

Remarque_-3 : $\frac{\partial J}{\partial}(u)$ est en général une fonction bien moins régulière que $a(u):$ on ne peut donc appliquer rigoureusement une méthode de gradient au cas continu ! 
Remargue 4 : $_{\text {d }} \frac{\partial^{J}}{\partial a}(u)$ est le gradient de J par rapport à la fonction a, sans aucune hypothèse sur la forme algêbrique de cette fonction a.

Mais si pour des raisons physiques ou pratiques, on désire chercher a(u) sous la forme :

$$
a(u)=\alpha\left(u, \beta_{1} \ldots \beta_{k}\right)
$$

où $\alpha\left(u, \beta_{1} \ldots \beta_{k}\right)$ est une fonction connue de $u$ et de $k$ paramètres inconnus $\beta_{1} \ldots \beta_{k}$, il est immédiat de calculer $\frac{\partial J}{\partial \beta_{j}}, j=1,2, k$ à partir de $\gamma(u)$ :

$$
\frac{\partial J}{\partial \beta_{j}}(\beta)=\int_{D} \gamma(u) \frac{\partial \alpha}{\partial \beta}\left(u, \beta_{1} \ldots \beta_{k}\right) d u \quad j=1,2 \ldots k
$$

Remarque_3 : Supposons qu'un progranme ait été écrit en vue de I'identification de a en tant que fonction de $u$, sans aucune spécification de forme algébrique. A chaque itération de gradient, le programme modifie le paramètre a suivant (1'équivalent discret de) la formule :

$$
a^{n+1}(u)=a^{n}(u)-\rho \frac{\partial J}{\partial a}\left(a^{n}\right)(u)
$$

Supposons que I'on désire modifier le programme pour chercher a sous la forme (19), où $\alpha$ est supposée être linéaire par rapport aux paramètres $\beta$. Il faut que $\beta$ soit modifié suivant le schëma :

$$
\beta^{n+1}=\beta^{n}-\rho \frac{\partial J}{\partial \beta}\left(\beta^{n}\right)
$$

Mais grâce à la linéarité de $\alpha$ par rapport à $\beta$, la forme (13) est strictement équivalente à :

$$
a^{n+1}(u)=a^{n}(u)-p \alpha\left(u, \frac{\partial J}{\partial \beta}\left(\beta^{n}\right)\right)
$$

où $a^{n}$ et $a^{n+1}$ sont de la forme (19).

En comparant (22) à (24), on voit que les seules modifications à faire dans le programme consistent à inséxer, juste après que la fonction $\frac{\partial J}{\partial a}\left(a^{n}\right)$ (u) ait été calculée :

- le calcul de $\frac{\partial J}{\partial \beta}\left(\beta^{\mathrm{n}}\right)$ par la formule (21) (quadratures)

- Le stockage, dans la mémoire assignée à $\frac{\partial J}{\partial a}\left(a^{n}\right)(u)$, de la fonction $\alpha\left(u, \frac{\partial J}{\partial \beta}\left(\beta^{n}\right)\right)$. 


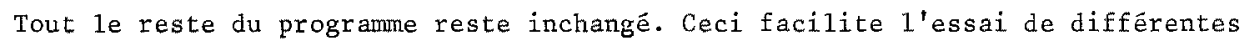
formes algébriques pour la fonction $a$.

Donnons maintenant les applications numériques, qui j'espère éclaireront les considérations précédentes.

IV - APPLICATIONS

Dans tout ce paragraphe :

$\Omega \subset \mathbb{R}$ ou $\mathbb{R}^{2}$ sera un domaine spatial borné, de froutière $\Gamma, \underline{x \in \Omega \text { sera } 1 a}$ variable d'espace,

]$_{\text {OT }}[\subset \mathbb{R}$ sera l'intervalle de temps sur lequel on observera les phénomènes dynamiques, $t \in]$ от [ ëtant la variable de temps,

$y \in \mathbb{R} \quad$ sera la variable dépendante (c.a.d. la solution des équations).

lère Application : Identification de la perméabilitê d'un aquifère dans la région de Bordeaux.

Nous voulons ajuster le modèle d'un aquifère statique dans un domaine carré à deux dimensions d'espace.

Les êquations du système sont :

$$
\left.\begin{array}{l}
-\frac{\partial}{\partial x_{1}}\left(a(x) \frac{\partial y}{\partial x_{1}}\right)-\frac{\partial}{\partial x_{2}}\left(a(x) \frac{\partial y}{\partial x_{2}}\right)=f(x) \text { dans } \Omega \\
y=g \text { sur } \Gamma
\end{array}\right\}
$$

où

- y est la pression de 1 'eau au point $x \in \Omega$

- $a(x)$ est la perméabilité inconnue de l'aquifère (supposé isotrope) au point $\mathrm{x}=\left(\mathrm{x}_{1} \mathrm{x}_{2}\right) \in \Omega$

- $f(x)$ est une fonction connue de $x=\left(x_{1}, x_{2}\right) \in \Omega$, liée au débit $q_{j}$ et à la position du jième puits par :

$$
f(x)=\sum_{j=1}^{43} q_{j} x_{j}(x)
$$

où $x_{j}(x)$ est une fonction caractérisant la géomêtrie et la position du $j^{\text {ième }}$ puits par : 


$$
x_{j}(x)=\left\{\begin{array}{l}
0 \text { si } x n^{\prime} \text { appartient pas au } j^{i e ̀ m e} \text { puits } \\
\text { une constante si } x \text { appartient au } j^{\text {ième }} \text { puits }
\end{array}\right.
$$

la constante étant choisie telle que :

$$
\int_{\Omega} x_{j}(x) d x=1
$$

- g est la pression connue sur la frontière $\Gamma$ de $Q$.

Nous supposons ici que nous avons une observation distribuée, c.a.d. que nous connaissons une fonction $z(x)$, qui est une mesure de la fonction $y(x)$. En pratique, dans le cas considéré ici, $z(x)$ a étẻ déduite par interpolation des isopiezes reprêsentêes sur la fig. 1 (mais on aurait pu éviter cette interpolation). La fonction $g$ est obtenue de la même façon. La fonction coût associée à a est alors :

$$
J(a)=\int_{\Omega}(y(x, a)-z(x))^{2} d x_{1} d x_{2}
$$

Nous allons maintenant faire rentrer ce problème dans le cadre théorique utilisé au §III. En remplaçant dans (25) y par $\mathrm{y}-\tilde{y}$, où ỹ est un relèvement de $\mathrm{g}$ dans $\boldsymbol{\chi}$, nous somnes ramenés à un système de la même forme que (25), mais avec $g=0$.

Nous supposons :

$$
a \in L^{\infty}(\Omega) \quad f \in L^{2}(\Omega)
$$

et nous identifions $L^{2}(\Omega)$ avec son dual, et $H_{0}^{1}(\Omega)$ à un sous espace de $L^{2}(\Omega)$. Alors $L^{2}(\Omega)$ peut être identifié à un sous espace du dual $\left[H_{0}^{1}(\Omega)\right]^{\prime}$ de $H_{0}^{1}(\Omega)$ :

$$
H_{0}^{1}(\Omega) \quad L_{L}^{2}(\Omega) \quad C\left[H_{0}^{1}(\Omega)\right]
$$

On peut alors associer à toute fonction a $\epsilon \mathrm{L}^{\infty}(\Omega)$ un opérateur linéaire $A(a)$ allant de $\mathrm{H}_{0}^{1}(\Omega)$ dans $\left[\mathrm{H}_{0}^{1}(\Omega)\right]$ ' par :

$$
(A(a) u, v)=\int_{Q} a(x) \sum_{i=1}^{\eta} \frac{\partial u}{\partial x_{i}} \frac{\partial v}{\partial x_{i}} d x \quad \forall u, v \in H_{0}^{1}(Q)
$$

On sait alors qu'une formulation faible de l'équation (25) (avec g=o) est donnée par : 


$$
\text { trouver } \begin{array}{ll} 
& y \in H_{0}^{1}(\Omega) \\
A(a) y=f & \text { tel que }
\end{array}
$$

L'équation (31) est l'équation d'êtat. Elle est de la forme (1) où $\mathrm{Y}=\mathrm{H}_{0}^{1}(\Omega), \quad Q=\mathrm{L}^{\infty}(\Omega)$ et $\mathrm{F}=\left[\mathrm{H}_{0}^{1}(\Omega)\right]^{\prime}$, et où

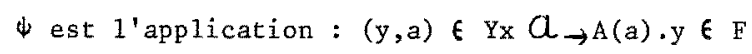

La partie $a_{c}$ de $a$ pour laquelle l'ëquation (31) admet une solution unique est ici :

$$
a_{c}=\left\{a \in L^{\infty}(\Omega) \mid \exists \alpha>0, a(x) \geqslant \alpha>o p \cdot p \cdot \operatorname{sur} \Omega\right\}
$$

L'ensemble $a_{a d}$ des paramètres admissibles introduits en (7) peut être défini ici par :

$$
a_{a d}=\left\{a \in L(\Omega) \mid \quad a_{\operatorname{Max}} \geqslant a(x) \geqslant a_{\min } p \cdot p \cdot \operatorname{sur} Q\right\}
$$

où $a_{\text {Max }}$ et $a_{\min }$ sont des bornes supërieures et inférieures connues des perméabilités.

En ce qui concerne 1'observation, nous prendrons ici :

$$
\mathcal{H}=\mathrm{L}^{2}(\Omega) \quad \mathscr{E}=\text { injection canonique de } \mathrm{E}_{0}^{1}(\Omega) \text { dans } \mathrm{L}^{2}(\Omega)
$$

de telle sorte que la fonctionnelle J dêfinie en (27) coïncide avec celle en (5).

On vêrifie facilement que les hypothèses de la proposition 2 sont satisfaites, et on obtient la

Proposition 3 : Sous les hypothèses (28), la fonctionnelle J définie en (27) est dêrivable par rapport à a $\in L^{\infty}(\Omega)$, et $J^{\prime}(a)$ est donnée par :

$$
J^{\prime}(a) \delta a=(A(\delta a) y, p) \quad \forall a \in a_{c}, \forall \delta a \in a
$$

où $p \in \mathrm{H}_{\mathrm{o}}^{1}(\Omega)$ est 1 'état adjoint, solution de

$$
A(a) p=-2(y-z)
$$

Compte tenu de (30), (37) se réécrit:

$$
J^{\prime}(a) \delta a=\int_{\Omega} \delta a(x) \sum_{i=1}^{2} \frac{\partial y}{\partial x_{i}} \frac{\partial p}{\partial x_{i}} d x \quad \forall \delta_{a \in L^{\infty}(\Omega)}
$$


ce qui prouve que la derivêe partielle fonctionnelle $\frac{\partial J}{\partial a}(x)$ existe et a pour expression :

$$
\frac{\partial J}{\partial a}(x)=\sum_{i=1}^{2} \frac{\partial y}{\partial x_{i}}(x) \frac{\partial p}{\partial x_{i}}(x) \quad \in L^{1}(Q)
$$

Cette formule nous permet de calculer numériquement aisèment $\frac{\partial J}{\partial a}$, lorsque nous connaissons $y$, solution de (31), c.a.d. solution faible de (25), et p, solution de (38), c.a.d. solution faible de :

$$
\left\{\begin{array}{l}
-\frac{\partial}{\partial x_{1}}\left(a\left(x_{1} x_{2}\right) \frac{\partial p}{\partial x_{1}}\right)-\frac{\partial}{\partial x_{2}}\left(a\left(x_{1} x_{2}\right) \frac{\partial p}{\partial x_{2}}\right)=-2\left(y\left(x_{1} x_{2}\right)-z\left(x_{1} x_{2}\right)\right) d a n s \\
p=0 \text { sur } \Gamma
\end{array}\right.
$$

Resultats numêriques: Les données (1) sont regroupées sur la figure 1.

Le domaine $\&$ était discrétisê en $20 \times 20$ points. La fontion inconnue $a\left(x_{1} x_{2}\right)$ était discrêtisée sur cette grille (il y avait donc 400 paramètres scalaires inconnus). A chaque itération de gradient, les équations (25) et (4l) étaient rësolues de façon classique par différences finies. Puis la fonction $\frac{\partial J}{\partial a}(x)$ était évaluée en chaque noeud de la grille par diffërences finies à partir de l'équation (40).

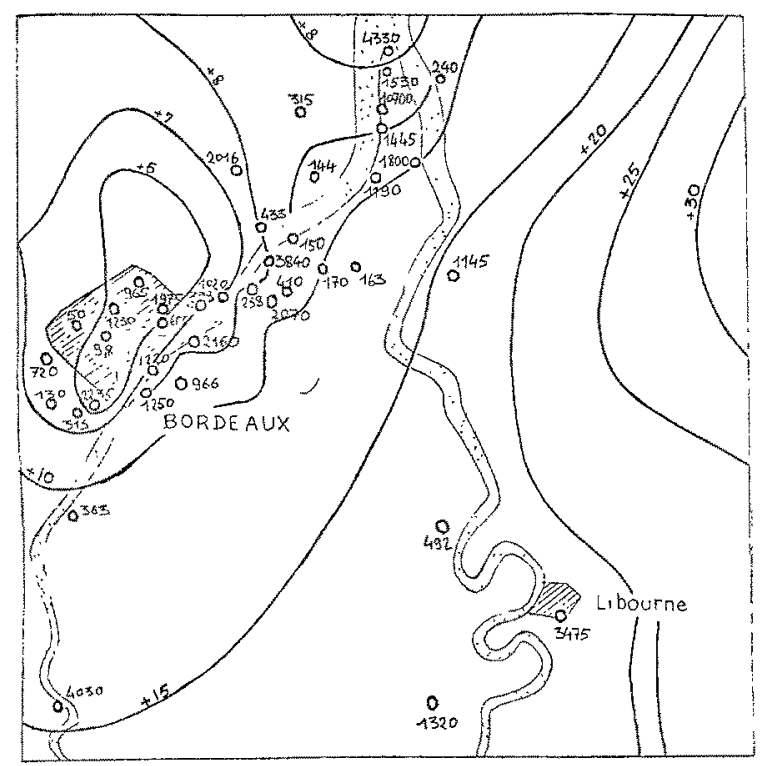

Fig. 1 : Les données du problème d'identification.

\footnotetext{
(1) Nous remercions le "Centre d'Informatique Géologique et Minière" de I'Ecole des "ines de Fontainebleau, qui nous a fourni ces données et nous a autorisé à les puhlier.
} 
Ensuite, un algorithme de plus grande descente était utilisẻ pour mettre à jour a avec une détermination approchée du $p$ optimal à chaque pas.

Comme nous n'avions aucune idée de l'ordre de grandeur de la perméabilité a, nous avons commencé par chercher $a(x)$ sous forme d'une constante (cf. Remarque 5). En partant de $a^{\circ}=150$, nous sommes ainsi arrivés à $a=1622$ en 9 itêrations de gradient et 25 s d'IBM 360-91.

Puis, partant de la fonction a(x) constante et égale à 1622 , nous avons obtenu la fonction a(x) représentée sur la figure 2 en 5 itërations de gradient et $22 \mathrm{~S}$ d'IBM $360-91$.

La pression y correspondant aux perméabilités $a\left(x_{1} x_{2}\right)$ de la figure 2 est représentée sur la figure 3 (à comparer avec la figure 1). 


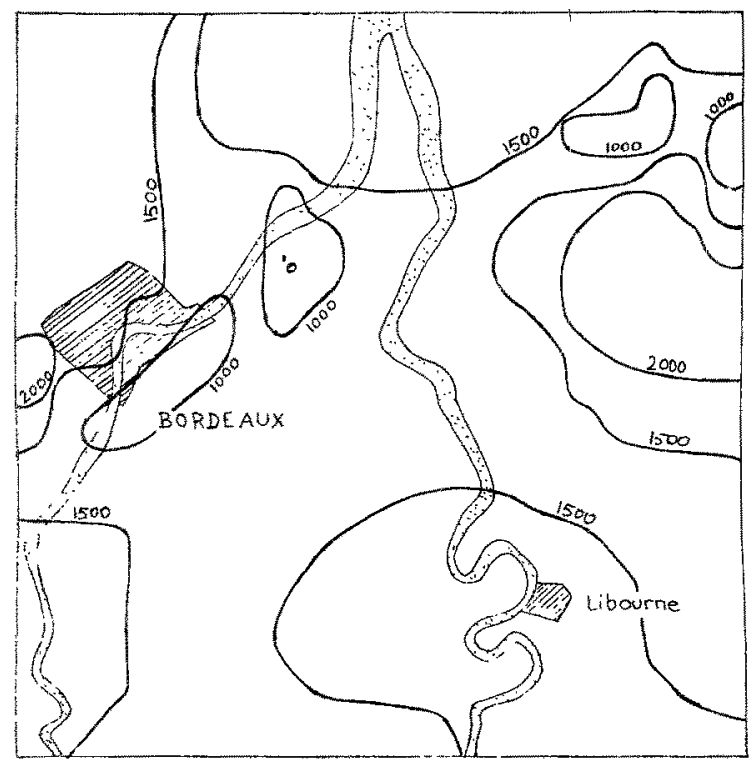

Fig. 2 : Carte des Transmissivités $a(x)$ fournies par l'algorithme.

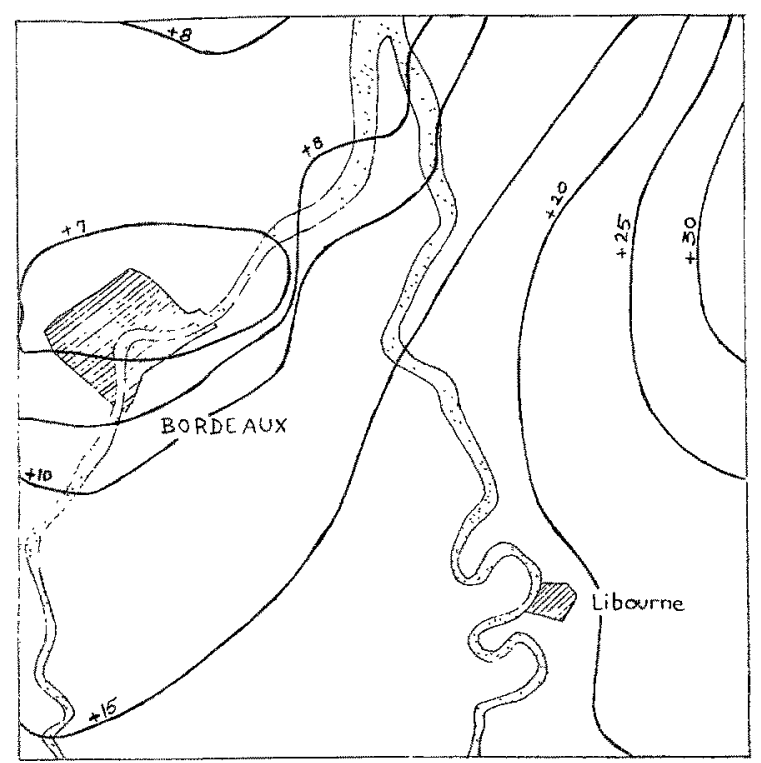

Fig. 3 : Carte des isopiezes calculées. 


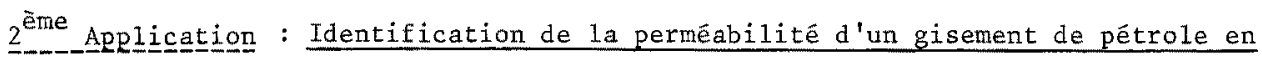
production monophasique.

Le gisement est le domaine \& représenté sur la fig. 4. L'équation d'état pour la pression $y(x, t)$ est :

$$
\left.\begin{array}{l}
\left.\frac{\partial y}{\partial t}-\sum_{i=1}^{2} \frac{\partial}{\partial x_{i}}\left(a(x) \frac{\partial y}{\partial x_{i}}\right)=f(x, t) \text { dans } Q x\right] \text { OT }[ \\
\frac{\partial y}{\partial n}=0 \text { sur } \Gamma \text { (condition de Neumann) } \\
y(x, o)=y_{0}(x) \text { sur } Q \text { (condition initiale) }
\end{array}\right\}
$$

où

a(x) représente la perméabilité inconnue du champs de pétrole, supposê isotrope,

$f(x, t)$ est une fonction connue de la forme (26), où les dêbits $q_{j}$ dépendent maintenant du temps,

$y_{0}(x, t)$ est la pression initiale dans le gisement, connue.

Nous disposong ici de moins de mesures de pression que dans l'exemple précêdent : seule la pression moyenne dans chacun des 11 puits est supposée mesurée à chaque instant $t(1)$, d'où onze fonctions du temps $z_{j}(t) j=1,2 \ldots 11$.

La fonction coût naturelle est ici :

$$
J(a)=\sum_{j=1}^{1} \int_{0}^{T}\left[\int_{\Omega} x_{j}(x) y(x, t ; a) d x-z_{j}(t)\right]^{2} d t
$$

De même que pour 1'exemple précédent, ce problème peut rentrer dans le cadre théorique du $\$ I I$, en utilisant la théorie variationnelle des équations aux dérivées partielles, et on peut appliquer la proposition 2. Pour plus de détail concernant. ces questions théoriques, cf. [3][4].

Les formules (13) et (12) deviennent :

$$
J^{\prime}(a) . \delta a=\int_{\Omega}\left[\int_{0}^{T}{ }_{i}^{2} \sum_{1} \frac{\partial y}{\partial x_{i}} \frac{\partial p}{\partial x_{i}} d t\right] \delta a(x) d x \forall \delta a \in L^{\infty}(\Omega)
$$

\footnotetext{
(1) En fait, c'est 1à une hypothèse irréaliste (il faut fermer le puits pour mesurer 1a pression...). La méthode s'adapte très facilement à des mesures de pressions discrêtes en temps, cf. [6].
} 


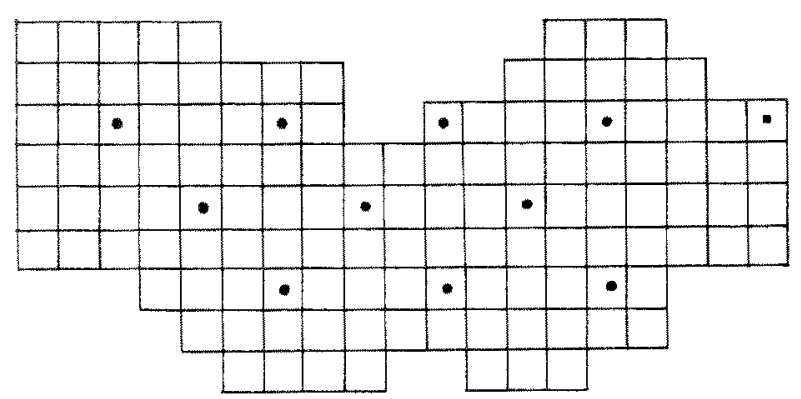

Fig. 4 : Discrétisation adoptée pour le gisement. Les points noirs reprësentent les puits de pétrole.

Ensuite, un algorithme de plů grande descente était utilisé pour mettre à jour a avec une détermination approchée du $\rho$ optimal à chaque pas.

Comme nous n'avions aucune idée de l'ordre de grandeur de la perméabilité a, nous avons commencé par chercher $a(x)$ sous forme d'une constante (cf. Remarque 5). En partant de $a^{\circ}=150$, nous sommes ainsi arrivés à $a=1622$ en 9 itérations de gradient et 25 s d'IBM 360-91.

Puis, partant de la fonction a $(x)$ constante et égale à 1622 , nous avons obtenu la fonction $a(x)$ représentée sur la figure 2 en 5 itérations de gradient et $22 \mathrm{~S}$ d'IBM 360-91.

La pression y correspondant aux perméabilités $a\left(x_{1} x_{2}\right)$ de la figure 2 est reprësentẻe sur la figure 3 (à comparer avec la figure 1). 


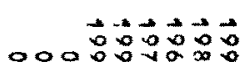

$000 \overrightarrow{0} \overrightarrow{0} \overrightarrow{0} \vec{\omega} \overrightarrow{0} \overrightarrow{0}$

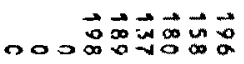

ND大ํํำ

$0000 \omega \rightarrow \tan$

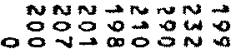

ÕO

$\mid \begin{aligned} & 100 \\ & 0 \\ & 0\end{aligned}$

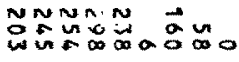

ONNNNVNW

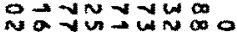

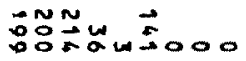

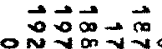

v2000

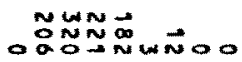

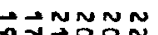

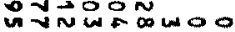

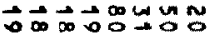

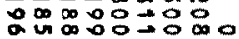

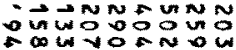

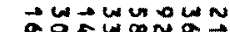

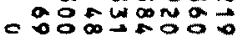

$\overrightarrow{0} \overrightarrow{N N}=N N$

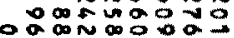

iี

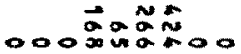

$000 \overrightarrow{00000} 00$

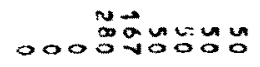

Nus $\overrightarrow{0} \overrightarrow{0} \overrightarrow{0}$ $0000^{\infty} 0000$

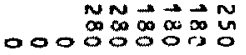

$N N N N \overrightarrow{N N}$

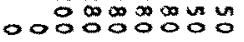
NNN

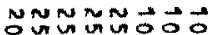

은

果

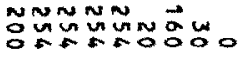

NNNNN

onvinnw

NNNN

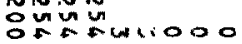

Nㅜㅇ

Oㅇㅇㅇㄴ 00

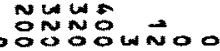

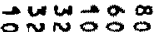

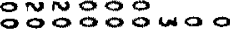

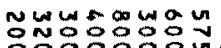

ww wawn w

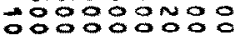

WN

- $\overrightarrow{0} \overrightarrow{0}$ wón

unan

000 ํํㅇㅇㅇำ

000붕영

$000 \overrightarrow{8}=\overrightarrow{5} \overrightarrow{0} 00$ 
(à comparer avec (17) et (39)) où y est la solution de (42), et où p (1'état adjoint) est solution de

$$
\left\{\begin{array}{l}
-\frac{\partial p}{\partial t}-\sum_{i=1}^{2} \frac{\partial}{\partial x_{i}}\left(a(x) \frac{\partial p}{\partial x_{i}}\right)=-2 \sum_{j=1}^{11} x_{j}(x)\left[\int_{\Omega} \chi_{j}(x) y(x, t) d x-z_{j}(t)\right] \\
\left.\frac{\partial p}{\partial n}=0 \text { sur } \Gamma \text { (condition de Neunann) dans } \Omega x\right] O T[ \\
p(x, T)=0 \text { sur } \Omega \text { (condition finale). }
\end{array}\right.
$$

Ainsi la formule (44) montre que la dérivêe partielle fonctionnelle $\frac{\mathrm{Q} J}{\mathrm{~J}}(\mathrm{x})$ existe dans cet exemple, et permet de la calculer aisèment à partir de $y$ et $p$.

Résultats numériques: Dans le cadre d'un contrat de recherche entre l'IRIALABORIA et I'IFP, cette théorie a étê appliquée à des données fournies par $1^{\prime}$ IFP (Institut Français du Pétrole). Nous remercions 1'IFP de l'autorisation de publier ces résultats.

Le gisement est inclus dans un rectangle de $8 \times 16,8 \mathrm{~km}$, et discrétisê en 127 mailles (le pas d'espace étant de $884 \mathrm{~m}$ ). L'historique de production (c.a.d. les fonctions $q_{j}(t)$ ) couvre 2070 jours (soit 5 ans) par pas de $23 j$. I1 est connu (bien que non représenté ici).

La pression initiale est connue, constante et égale à $482 \mathrm{~kg} / \mathrm{cm}^{2}$. Les mesures de pression $z_{j}(t)$ sont simulées en intégrant l'équation (42) avec les perméa bilitês "exactes" de la figure 5. En outre, les perméabilités exactes sont connues dans toutes les mailles contenant des puits. Nous pouvons prendre en compte cette information très facilement.

La valeur initiale des perméabilités a ëté choisie ëgale à 200 mdm partout, sauf dans les mailles contenant un puits, où la valeur exacte et connue fut imposée.

A chaque itération, les systèmes (42) et (45) étaient résolus par un schéma aux diffërences finies classique (une simulation durait $0,5 \mathrm{~s}$ de CDC 7600), puis $\frac{\partial J}{\partial a}$ était évalué en chaque noeud par (44), puis mis à zéro pour tous les noeuds où se trouvait un puits.

Les perméabilitês obtenues après 12 itérations de gradient et 50 s de CDC 7600 sont indiquées figure 6 . L'erreur quadratique moyenne sur la pression sur tous les puits était de $1,19 \mathrm{~kg} / \mathrm{cm}^{2}$.

Les pressions calculées et observées sont représentées, pour les deux puits ayant le meilleur et le plus mavais êcart quadratique moyen, sur les figures 7 et 8 . 


\section{WELL 1 .ITERATION 12}

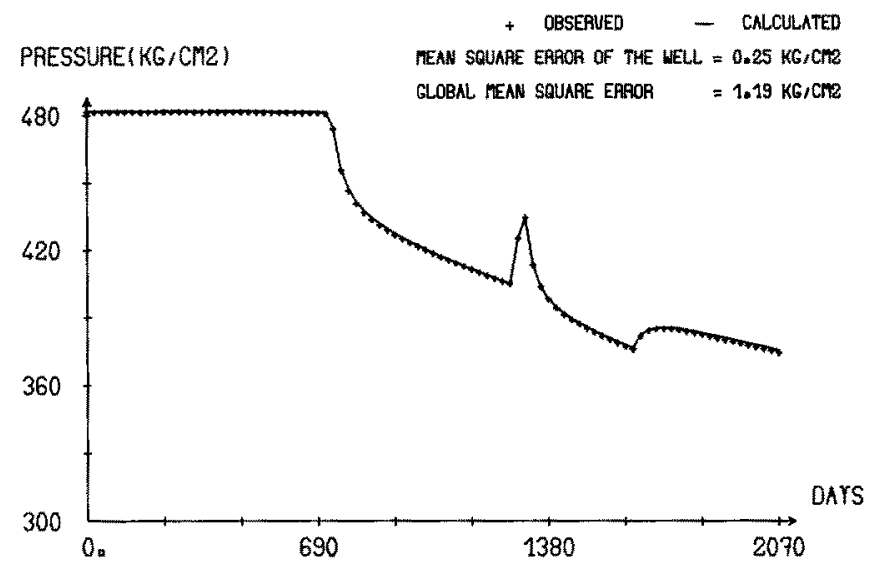

Fig. 7 : Pressions mesurêes

et calculées au puits

numéro 1.

WELL 10a ITERATION 12

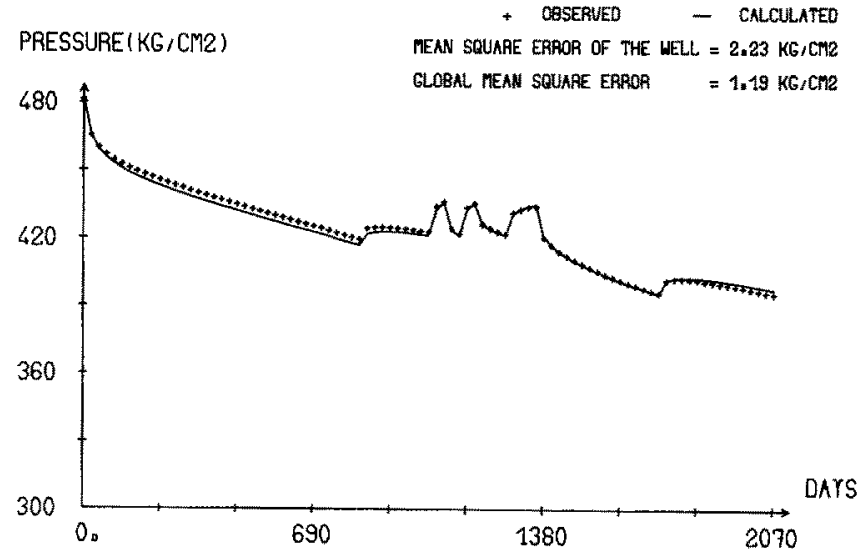

Fig. 8 : Pressions mesurêes et calculées au puits numéro 10 . 
En comparant les ígures 5 et 6 , on constate que les perméabilités obtenues sont assez différentes des perméabilités "exactes".

Ceci provient de ce qu'il n'y a aucun résultat d'unicité de la solution du problème inverse dans notxe exemple. Dans certaines régions, a(x) est resté prês de sa valeur initiale de $200 \mathrm{mdm}$ (en particulier sur la frontière et dans les zones où il y a peu de puits actifs) : ceci signifie simplement que les perméabilitês, dans ces reégions ont peu d'influence sur les mesures de pressions, et donc ne peuvent pas âtre déterminées à partir de ces mesures.

Dans d'autres régions (en particulier dans les zones où figurent des puits actifs) on constate que les perméabilitēs se sont déplacées dans la bonne direction, et que leur ordre de grandeur correspond bien à celui des perméabilités exactes.

\section{3 ème Application: Identification de la non-linéarité dans une equation parabolique} quasi linéaire.

Considérons, sur le domaine spatial $Q=$ ] 01 [, l'équation parabolique suivante :

$$
\left\{\begin{array}{l}
\left.\frac{\partial y}{\partial t}-\frac{\partial}{\partial x}\left(a(y) \frac{\partial y}{\partial x}\right)=f(x, t) \text { dans } \Omega x\right] \text { or }[ \\
y=g \operatorname{sur} \Gamma x] 01[\quad \text { (conditions de Dirichlet) } \\
y(x, o)=y_{0}(x) \text { sur } \Omega \text { (conditions initiales). }
\end{array}\right.
$$

Les fonctions $\bar{f}$, g et $\mathrm{y}_{\mathrm{o}}$ sont supposées connues, et nous voulons estimer la fonction $y \rightarrow a(y)$. Le système (46) peut reprêsenter un processus de diffusion de chaleur mono dimensionnel avec une conductibilitë thermique dëpendant de la température.

La solution $y(x, t)$ de (46) est mesurée, à chaque instant $t \in]$ oT [ , en 5 points $x_{1} \ldots x_{5}$ de 1 intervalle $] 01[$. On obtient ainsi cinq fonctions du temps $z_{1}(t) \ldots z_{5}(t)$.

La fonction coût est naturellement :

$$
J(a)=\sum_{j=1}^{5} \int_{0}^{T}\left(y\left(x_{j}, t ; a\right)-z_{j}(t)\right)^{2} d t
$$

D'un point de vue thëorique, ce problème est plus difficile que les deux précêdents, car 1'équation (46) n'est pas linéaire. En particulier, nous ne savons pas faire rentrer ce problème dans le cadre général du \$III. On peut cependant démontrer directement (cF. [7]) que la fonctionnelle J est Fréchet-dérivable. Nous ne donnons ici que les résultats fornels, sans préciser la nature de l'espace des parametres $a$ et de 1 'espace des solutions $Y$.

on peut donc montrer que : 


$$
\left\{\begin{array}{l}
\forall \delta a \in a \\
J^{\prime}(a) \delta a=\iint_{\Omega}^{T} \delta a(y(x, t)) \frac{\partial y}{\partial x}(x, t) \frac{\partial p}{\partial x}(x, t) d x d t
\end{array}\right.
$$

où $y(x, t)$ est $1 a$ solution de $(46)$, et $p(x, t)$ est 1'état adjoint, solution de

$$
\left\{\begin{array}{l}
-\frac{\partial p}{\partial t}-a(y(x, t)) \frac{\partial^{2} p}{\partial x^{2}}=-2 \sum_{j=1}^{5} \delta\left(x-x_{j}\right)\left(y\left(x_{j}, t\right)-z_{j}(t)\right) \\
p=0 \text { sur } r \quad x] \partial T[ \\
p(x, T)=0 \quad \text { (condition finale). }
\end{array}\right.
$$

En posant :

$$
\left.\varphi(x, t)=\frac{\partial y}{\partial x}(x, t) \frac{\partial P}{\partial x}(x, t) \quad \forall x, t \in \Omega x\right] \text { oT }[
$$

1 'ëquation (49) devient :

$$
\left\{\begin{array}{l}
\forall \delta a \in a \\
J^{\prime}(a) \cdot \delta_{a}
\end{array}=\int_{\Omega} \int_{0}^{T} \delta a(y(x, t)) \varphi(x, t) d x d t\right.
$$

En comparant (51) avec (17) qui donne la forme canonique de J'(a). $\delta_{a}$, nous constatons que (51) ne nous donne pas la dérivée partielle fonctionnelle $\gamma(z)$ de $J$ par rapport à $a(z)$, définie par

$$
\left\{\begin{array}{l}
\forall \delta_{a} \in a \\
J^{\prime}(a) . \delta a=
\end{array} \int_{y_{m}}^{y_{M}} \delta a(z) y(z) d z\right.
$$

où $y_{m}$ et $y_{M}$ sont des bornes inférieures et supérieures connues de la solution y de $(46)$.

Nous distinguerons ici deux cas:

er cas : Si nous supposons que a(y) est une fonction de forme connue, avec seulement un nombre fini de paramètres inconnus $\beta_{1} \ldots \beta_{k}$ (cf. remarque 4 , où $x=y$ ). Il est alors facile de calculer le gradient de J par rapport à $\beta$ à partir de (51) :

$$
\frac{\partial J}{\partial \beta_{j}}(\beta)=\int_{\Omega} \int_{0}^{1} \frac{\partial \alpha}{\partial \beta}\left(y(x, t), \beta_{1} \ldots \beta_{k}\right) \varphi(x, t) d x d t
$$


Ainsi lorsque la fonction $\varphi(x, t)$ a été calculée numériquement (ce qui suppose que les deux systèmes (46) et (49) ont été résolus), le calcul du gradient de J par rapport à $\beta$ ne nécessite que $k$ quadratures. En outre la remarque 5 s'applique ici avec de légères modifications.

$$
\begin{aligned}
& \begin{array}{l}
2_{\text {ème }} \text { cas }: \text { Nous ne supposons aucune forme algébrique pour la fonction } \\
y \rightarrow a(y): \text { il nous faut donc trouver une façon de mettre } \\
(51) \text { sous la forme }(52) \text {. }
\end{array} \\
& \text { Définissons, pour tout nombre réel } \zeta: \\
& \qquad Q_{\zeta}=\{(x, t) \in \Omega x] 01[\mid y(x, t) \geqslant \zeta\}
\end{aligned}
$$

et

$$
\eta(\zeta)=\int_{\zeta} \varphi(x, t) d x d t
$$

La formule (51) devient alors :

$$
\int \begin{aligned}
& \forall \delta a \in a \\
& J^{\prime}(a) \delta a
\end{aligned}=\int_{y_{m}}^{y_{M}} \delta a(\zeta) d \eta(\zeta)
$$

Ce qui, en comparant à (52) donné :

$$
\frac{\partial y}{\partial a}(a)(y)=\gamma(y)=\eta^{\prime}(y)
$$

Ces formules nous permettent donc de calculer la dérivée partielle fonctionnelle de $J$ par rapport à la fonction $y \rightarrow a(y)$.

\section{Résultats numériques :}

Le domaine $\Omega=101$ [ était discrétisé en 20 intervalles de longueur 0,05 , et 1'intervalle ]OT[ en 40 pas de longueur 0,025. La fonction fêtait une combinaison linêaire de masses ia dirac discrêtes aux points $x_{1}=0,1 \quad x_{2}=0,3 \quad x_{3}=0,5 \quad x_{4}=0,7$ $x_{5}=0,9$.

La valeur initiale de y était constante, et la fonction exacte a $a_{0}(y)$ utilisée pour simuler les données était :

$$
a_{0}(y)=0,21-0,28 y+0,7 y^{2}
$$

Les bornes inférieures et supérieures de y choisies étaient :

$$
y_{m}=0,3 \quad y_{M}=2
$$




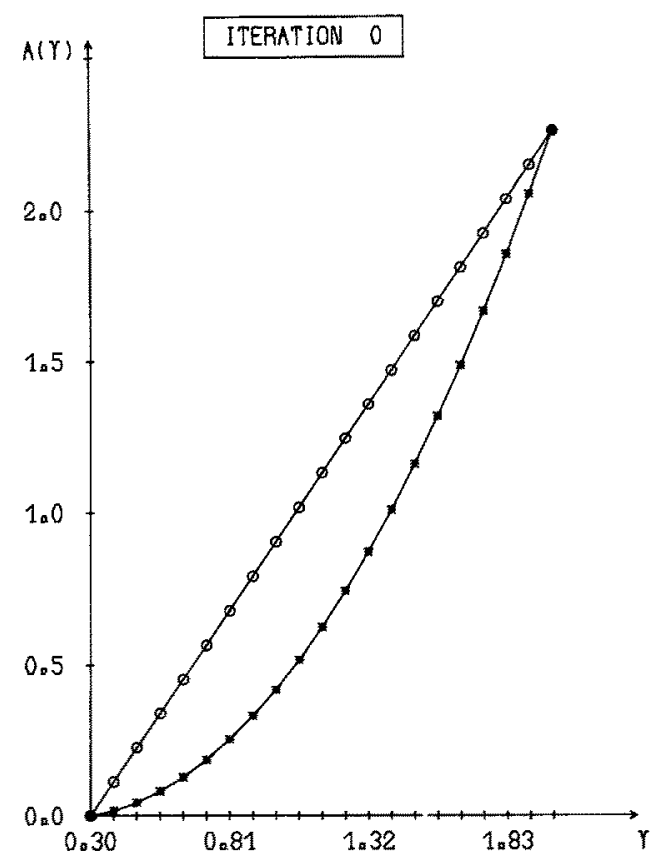

Fig. 9 : Valeur initiale et valeur exacte de la fonction a(y).

* fonction exacte

- FONCTION IDENTIFIEE

Fig. 10 : Recherche sous forme d'un polynôme du 2ề degrê.. Fonction obtenue après 4 itérations.

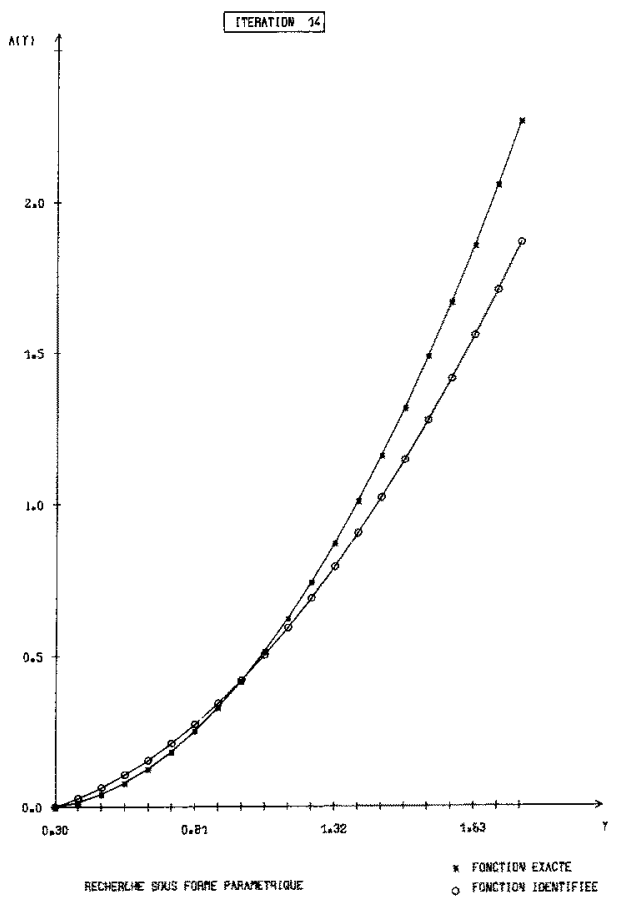




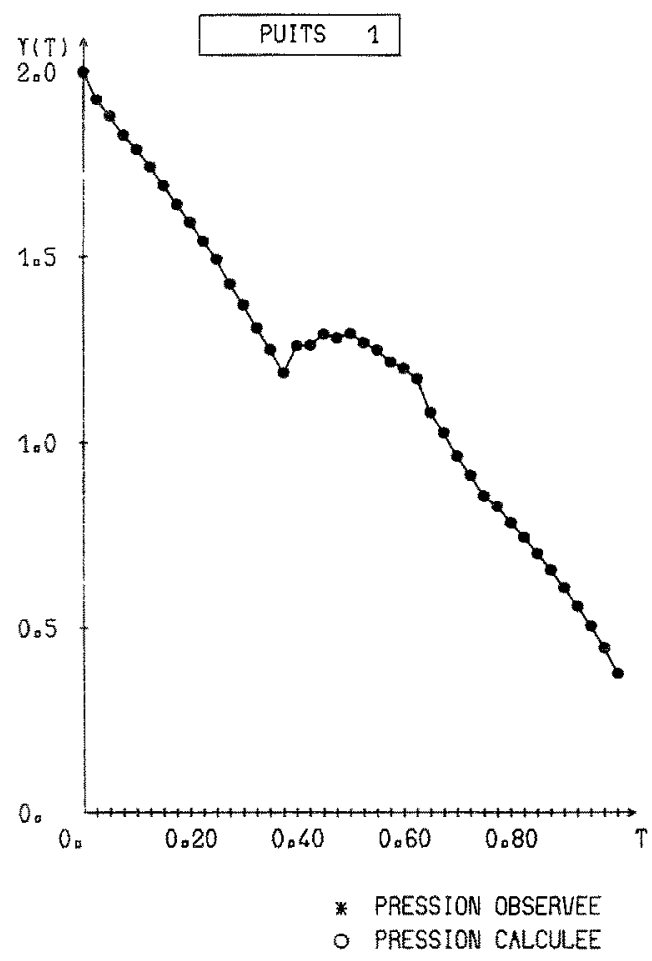

Fig. 11: Pressions mesurées et calculêes au puits 1 . 
et l'intervalle $] \mathrm{y}_{\mathrm{m}}, \mathrm{y}_{\mathrm{M}}[$ était divisë en 20 sous-intervalles de longueur $\Delta$ :

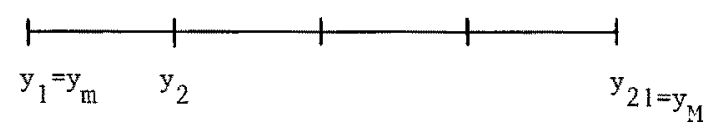

et la fonction a(y) était représentée sur cet intervalle par une fonction continue linéaire par morceaux :

$$
a(y)=\sum_{j=1}^{20}\left(a_{j}+\frac{1}{\Delta}\left(y-y_{j}\right)\left(a_{j+1}-a_{j}\right)\right) x_{j}(y)
$$

otì :

$$
\left\{\begin{array}{l}
x_{j}(y)=\left\{\begin{array}{l}
1 \text { si } y \in\left[y_{j}, y_{j+1}[\right. \\
0 \text { sinon }
\end{array}\right. \\
a_{j}=\text { représentation discrète de } a\left(y_{j}\right) .
\end{array}\right.
$$

Le système êtait ensuite intégré avec la valeur "exacte" a de la fonction a, par un schëma aux différences finies du type prédicteur-correcteur, ce qui fournissait les observations $z_{j}(t) j=1,2 \ldots 5$.

Pour retrouver la fonction $a(y)$, nous utilisons ensuite une mêthode classique de gradient (steepest-descent) avec détermination approchée du $\rho$ optimal et projection sur un polyedre convexe $a_{a d}$.

Tout d'abord, nous avons cherchẻ a (y) sous forme d'un polynôme de degrế 2 (3 inconnues scalaires), le gradient par rapport à 3 coefficients ètait calculé par (53).

Comme la fonction a doit être positive (si 1 'on veut que 1'équation (46) ait une solution !) l'ensemble convexe $a_{a d}$ de $R^{3}$ fut déterminé en êcrivant que, en chaque point de discrétisation $y_{j} \in\left[y_{m}, y_{M}\right]$, le polynôme a( $\left.y_{j}\right)$ devait être positif ( 21 contraintes linéaires).

La valeur initiale et les fonctions $a(y)$ obtenues après 14 itêxations de gradient sont montrées sur les figures 9 et 10. Après 32 itérations, la fonction obtenue est identique à la fonction exacte (58). Les sorties calculées et mesurées correspondantes sont montrées pour 1 e point $\mathrm{x}_{1}$ sur la figure 11 . 
Nous avons ensuite cherché $a(y)$ sous la forme $(60)$ d'une fonction continue linéaire par morceaux (21 paramètres inconnus).

La dérivêe $\frac{\partial^{J}}{\partial^{a}}$ est calculée à partir de (57) pour chaque $j$, ce qui nécessite une intégration nuthérique d'une fonction (liêe à la fonction $\varphi$ définie en (50)) sur le domaine $Q_{y_{j+1}}-Q_{y_{j}}$ pour chaque $j$.

i) Nous avons d'abord supposé que nous ne connaissions rien sur a, si ce n'est que c'est une fonction positive (a est une fonction positive "libre"):

$$
\left.a_{a d}=|a| a_{j} \geqslant 0 \quad j=1,2 \ldots 21\right\}
$$

Il est facile de projeter sur ce convexe !

La valeur initiale était celle de la figure 9, et la fonction a(y) obtenue après 20 itérations de gradient est montrée sur la figure 12 .

ii) Nous avons ensuite supposé que nous avions quelques informations sur la dérivêe seconde de 1 a fonction $y \rightarrow a(y):$

$$
a_{a d}=\left\{a \mid a_{j} \geqslant 0, j=1,2 \ldots 21, \quad-\underset{j}{j} \leqslant \frac{a_{j+1}-2 a_{j}+a_{j-1}}{\Delta^{2}} \leqslant M_{j}\right.
$$

où les $\mathrm{m}_{j}$ et $\mathrm{M}_{\mathrm{j}}$ sont des nombres positifs domés. Des informations de ce genre sont souvent connues des physiciens (ils peuvent connaître le sens de la concavitê d'une non linëarité par exemple), et nous pouvons ici les prendre en compte facilement.

Les résultats correspondant à la valeur initiale de la figure 9 et à différents $m_{j}$ et $M_{j}$ sont représentés sur les figures 13 et 14 . 

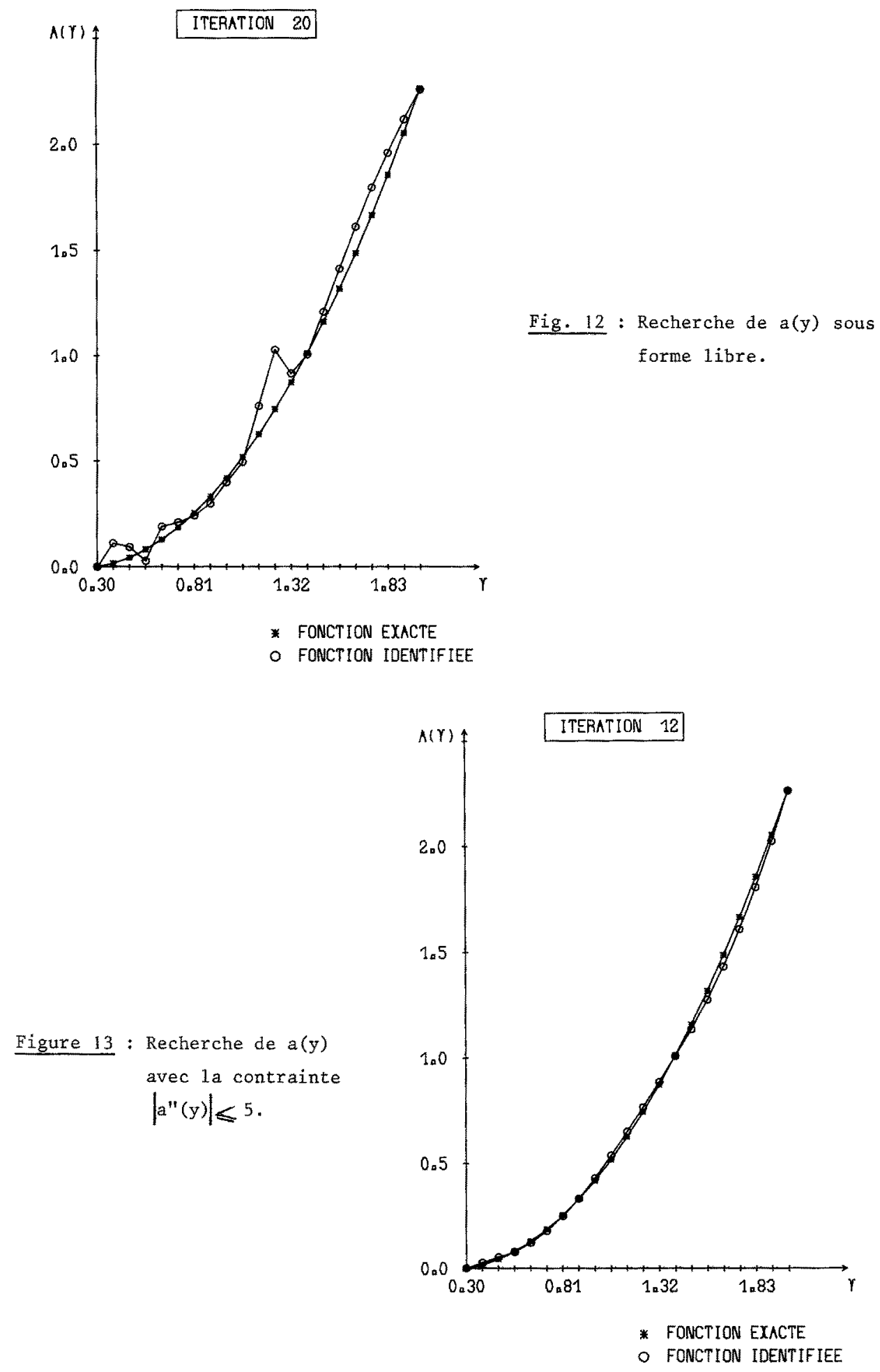


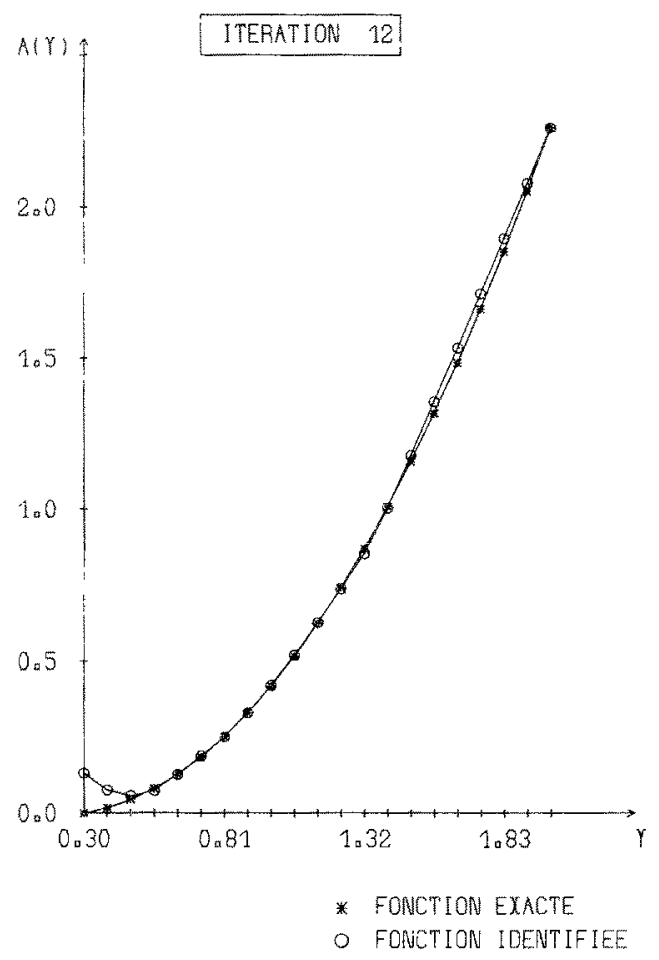

Fig. 14 : Recherche de $a(y)$ avec la contrainte $0 \leqslant a^{\prime \prime}(y) \leqslant 5$. 
$\mathrm{V}-$ CONCLUSION

Nous avons essayê de convaincre le lecteur qu'il est possible, lorsqu'on rencontre des fonctions inconnues dans la modélisation d'un système, d'éviter toute hypothèse sur la forme algébrique de cette fonction, et ceci jusqu'au traitement numérique, en utilisant la dérivée partielle fonctionnelle.

En outre, dans le cas où l'on recherche une fonction d'une forme algëbrique donnée, la dérivée partielle fonctionnelle est un intermédiaire très souple et permettant de changer de forme algëbrique à moindre frais.

I'utilisation de l'opérateur d'observation 6 , dont on demande seulement 1a dérivabilitë, nous permet de prendre en compte les mesures éfectuées sur le système sans avoir à leur faire subir de traitement préalable important.

Cette approche est systèmatique, et peut être appliquée à de nombreux autres domaines. De nombreux exemples sont étudiés dans [5].

VI - REFERENCES

[1] LIONS J.L. Contrôle optimal de systèmes gouvernếs par des équations aux dérivées partielles. Dunod 1968.

[2] LIONS J.L. Quelques mêthodes de résolution de problèmes aux limites non linéaires, Dunod Gauthier Villars Paris 1969.

[3] Chavent G. Sur une Méthode de résolution du Problème inverse dans les Equations aux dêrivées partielles paraboliques. Note CRAS Paris, T.260 Déc, 1969.

[4] CHAVENT G. Deux résultats sur le problème inverse dans les équations aux dérivées partielles du $2^{\text {ème }}$ ordre en $t$ et sur 1 'unicité de la solution du problème inverse de la diffusion. Note CRAS. Paris t. 270 , Janvier 1970 .

[5] CHAVENT G. Analyse fonctionnelle et Identification de coefficients répartis dans les équations aux dérivées partielles. Thèse d'Etat. Paris 1971 . 
[6] CHAVENT G., DUPUY \%., LEMONNIER P. History Matching by use of optimal control theory. Full Meeting of SPE, Las Vegas 1973.

[7] CHAVENT G., LEMONNIER P. Identification de la non linéarité dans une une équation parabolique quasi linéaire. A paraître đans "Applied Mathematics and Optimization, an international Journa1". 